\title{
SIMULAÇÃO DO PROCESSO DE FABRICAÇÃO DE MÁSCARAS DE TECIDO EM UMA CONFECÇÃO UTILIZANDO O SOFTWARE ARENA
}

\author{
Lucas da Silva Vieira -FIC/UNIS \\ lucas.dasilvavieira@alunos.unis.edu \\ Larissa Máximo Gonçalves -FIC/UNIS \\ larissa.goncalves@alunos.unis.edu.br \\ QuérenHapuquedeSouza Eugênio-FIC/UNIS \\ queren.eugenio@alunos.unis.edu.br \\ Vitória Durães - FIC/UNIS \\ Vitória.andrade@alunos.unis.edu.br \\ Tiago Bittencourt Nazare-FIC/UNIS \\ tiago@unis.edu.br
}

\section{RESUMO}

O presente artigo tem por objetivo descobrir através de técnicas de simulação computacional a capacidade de produção de uma confecção de vestuário no processo de fabricação de máscaras de tecido, seus gargalos e filas e o grau de ocupação dos recursos disponíveis. A partir da coleta de dados, feita pelo processo de cronoanálise, por meio de visitas a empresa, a modelagem computacional foi desenvolvida através dosoftware Arena versão Student 12, onde foi possível identificar a capacidade produtiva, em quais setores ocorrem os possíveis atrasos na produção (gargalos e filas) e o grau de ocupação. Para a elaboração do processo estatístico dos dados foi utilizado o software Minitab.

Palavras-Chave:Simulação, Processo, Fabricação, Confecção

\section{ABSTRACT}

Thisarticleaimstodiscover, theproductioncapacityof thefabricmaskmanufacturingprocess, a throughcomputersimulationtechniques, garmentmanufacture in its

bottlenecksandrowsandthedegreeofutilizationoftheavailableresources. Fromthe 
data collection, madebythechronoanalysisprocess, throughvisitstothecompany, a computationalmodelwasdevelopedusingthe software Arena versionStudent 12, where it waspossibletoidentifytheproductioncapacity, in whichsectorsthefactors are behind In production (bottlenecksandqueues) andthedegreeofoccupation. Toelaboratethestatistical data processused in theMinitab software.

Keywords:Simulation, Process, Manufacturing, Manufacturing

\section{INTRODUÇÃO}

As indústrias têxteis e de confecção segundo Lidório (2008), estão entre as atividades fabris mais antigas na história da humanidade. Atualmente, fazem usode altas tecnologias, métodos e processos bastante conhecidos. Normalmente sãouma das primeiras atividades industriaisa serem instaladas em um local e é uma grande absorvedora de mãodeobra.

O segmento têxtil, incluindo confecções e vestuário, possui grande relevância na economia nacional e local, sendo forte gerador de postos de trabalho (ARAÚJO; PEREIRA, 2006).

Piazzarolloet al., (2008), salienta a importância de se ter um sistema produtivo adequado, de forma a evitar muitas perdas ao longo processo.

De acordo com Campos (2006), a simulação consiste na reprodução de um processo real para um sistema artificial e, por meio de análises deste sistema são emitidos os resultados a respeito das particularidades de seu funcionamento. Oliveira (2012), aponta que o processo de simulação é um instrumento utilizado para estudar o funcionamento de um processo por intermédio da modelagem. $O$ modelo ilustra os elementos do sistema que contribuem para o entendimento do processo real.

Segundo Gonçalves (2004), é recomendado à utilização da técnica de simulação, pois a interferência em um sistema real poderá incidir na geração de um custo elevado, além de grandes riscos para o processo. 
O presente artigo tem por objetivo descobrir através de técnicas de simulação computacional no software Arena versãoStudent 12,a capacidade produtiva no processo de fabricação de máscaras de tecido, seus gargalos e filas e o grau de ocupação dos recursos disponíveis.

\section{REFERENCIAL TEÓRICO}

\subsection{Simulação computacional}

A simulação segundo Pegdenet al., (1995), consiste no método de desenvolver um modelo computacional de um sistema real e proporcionar experimentações com este modelo, com a finalidade de compreender o seu comportamento e/ou analisar as estratégias de seu funcionamento.

De acordo com Freitas Filho (2008), a simulação, sobretudo a computacional, vem sendo cada vez mais utilizada como metodologia,possibilitando aos gestores buscar ou encaminhar soluções aos problemas com que lidam frequentemente.

Para Gomes (2009), a simulação nada mais é do que uma maneira de fazer experimentos através de um modelo, verificando como o sistema responderá mediante as modificações que lhe são propostas. Para cada alteração realizada, é possível visualizar os resultados e analisar o novo desempenho do sistema, a partir dos relatórios gerados pelo softwareutilizado.

De acordo com Harrel et al., (2002), uma das principais vantagens da simulação, é a possibilidade de fazer testes sem interromper ou perturbar o sistema real. 


\subsection{Software ARENA}

O software de modelagem Arena é um ambiente gráfico integrado de simulações, que contém inúmeros recursos para modelagem, animação, análise estatística e análise de resultados (SANTOS e BEM, 2013).

O Arena é um software usado com finalidade de modelar os mais diversos ambientes, simulando um grupo de atividades utilizadas na fabricação de algum item, na prestação de serviços, dentre outros (SILVA et al., 2015).

De acordo com autor Fioroni, (2007), o funcionamento de uma modelagem no Arena ocorre da seguinte maneira, no decorrer da elaboração do modelo, o usuário expõe todas as partes estáticas como recursos e outros, bem como as especificações a serem seguidas. Ao se iniciar a simulação, os elementos dinâmicos (entidades) entram no modelo, interagem com os elementos estáticos e circulam conforme as regras que foram modeladas.

\section{MATERIAS E MÉTODOS}

A presente pesquisa se caracteriza como sendo um estudo de caso realizado em uma confecção localizada no município de Cataguases, Minas Gerais.Segundo Yin (2015), o estudo de caso é caracterizadocomo um método de pesquisa empregado em inúmeras situações,afim de colaborar para o conhecimento de pessoas ou grupos, onde manifestao propósito de compreender os fenômenos sociais complexos. Possibilita aos pesquisadores condicionar as características holísticas e significativas da realidade, tais como agestão de processos e organizacionais, eficiência, comportamento de pequenos grupos, dentre outros.

A empresa objeto de estudo está atuando no mercado de confecção de vestuário há mais de uma década, tendo uma área fabril de aproximadamente $200 \mathrm{~m}^{2}$. Atualmente, devido a pandemia da Covid-19, a empresa precisou ajustar seu portfólio de produtos, incrementando a fabricação de máscaras de tecido. Atualmente esse novo item representa o maior volume de produção.Além dos 
colaboradores, a empresa ainda conta com um sócio/gerente que éresponsável por toda administração.Na figura 1, pode-se observar a composição macro do processo produtivo da confecção.

Figura 1: Processo produtivo da empresa.

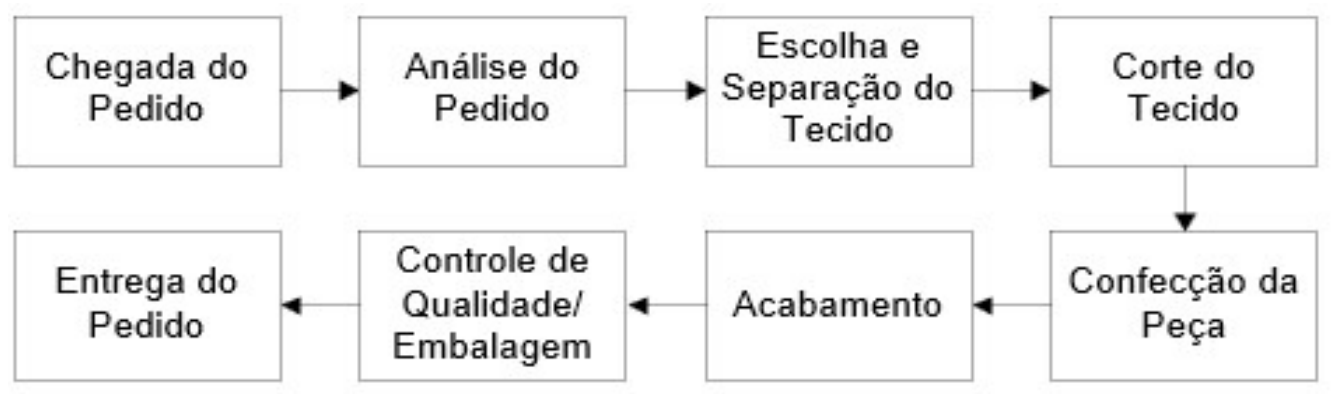

Fonte:Próprio Autor

As pesquisas bibliográficas existentes no trabalho foram efetuadas por meio de livros, no Google Acadêmico, anais de congressos, entre outras fontes eletrônicas.

Para a realização desta pesquisa, foram feitas visitas à empresa, análises do local de trabalho e das atividades desempenhadas durante o expediente. Entre os dias 01 a 15 de junho de 2020, foram coletadas 2 amostras diárias de cada etapa do processo de fabricação objeto de estudo.

Para mensurar os tempos foiusado a cronoanálise, que segundo Reis et al., (2015), contribui na determinação dos tempos de realização da tarefa, reduzindo a fadiga e extinção da ociosidade.

De acordo com Laugeni e Martins (2010) na prática deverá ser efetuada entre 10 e 20 tomadas de tempo dos processos. 
$\mathrm{Na}$ coleta dos tempos foi utilizado cronômetro digital da marca Kalenji e modelo Onstart 710, folhas A4 para anotações, uma prancheta e canetas esferográficas. As amostras dos tempos do processo estão disponíveis no quadro1, afim de certificar que as amostras coletadas representam a realidade do processo objeto de estudo

Quadro 1: Tempos dos processos (em segundos) de cada etapa produtiva

\begin{tabular}{|c|c|c|c|c|c|c|}
\hline $\begin{array}{c}\text { Processo } \\
\text { IDia }\end{array}$ & Corte & Unir Frente & UnirForro & $\begin{array}{c}\text { Unir Frente } \\
\text { e Forro }\end{array}$ & $\begin{array}{c}\text { Pregar } \\
\text { Elástico }\end{array}$ & Embalar \\
\hline 1 & $00: 00: 59$ & $00: 00: 35$ & $00: 00: 39$ & $00: 03: 22$ & $00: 00: 24$ & $00: 00: 14$ \\
\hline 1 & $00: 00: 55$ & $00: 00: 37$ & $00: 00: 40$ & $00: 03: 38$ & $00: 00: 22$ & $00: 00: 12$ \\
\hline 2 & $00: 00: 54$ & $00: 00: 33$ & $00: 00: 35$ & $00: 03: 54$ & $00: 00: 27$ & $00: 00: 10$ \\
\hline 2 & $00: 00: 57$ & $00: 00: 35$ & $00: 00: 36$ & $00: 03: 49$ & $00: 00: 29$ & $00: 00: 12$ \\
\hline 3 & $00: 00: 56$ & $00: 00: 34$ & $00: 00: 37$ & $00: 03: 58$ & $00: 00: 24$ & $00: 00: 14$ \\
\hline 3 & $00: 00: 55$ & $00: 00: 33$ & $00: 00: 36$ & $00: 03: 52$ & $00: 00: 26$ & $00: 00: 13$ \\
\hline 4 & $00: 00: 52$ & $00: 00: 38$ & $00: 00: 39$ & $00: 03: 40$ & $00: 00: 27$ & $00: 00: 11$ \\
\hline 4 & $00: 00: 53$ & $00: 00: 35$ & $00: 00: 36$ & $00: 03: 51$ & $00: 00: 23$ & $00: 00: 14$ \\
\hline 5 & $00: 00: 57$ & $00: 00: 32$ & $00: 00: 38$ & $00: 04: 02$ & $00: 00: 25$ & $00: 00: 11$ \\
\hline 5 & $00: 00: 55$ & $00: 00: 35$ & $00: 00: 37$ & $00: 03: 51$ & $00: 00: 24$ & $00: 00: 10$ \\
\hline 6 & $00: 00: 54$ & $00: 00: 40$ & $00: 00: 34$ & $00: 03: 59$ & $00: 00: 25$ & $00: 00: 13$ \\
\hline 6 & $00: 00: 57$ & $00: 00: 35$ & $00: 00: 36$ & $00: 04: 06$ & $00: 00: 26$ & $00: 00: 11$ \\
\hline 7 & $00: 00: 56$ & $00: 00: 38$ & $00: 00: 38$ & $00: 03: 45$ & $00: 00: 23$ & $00: 00: 14$ \\
\hline 7 & $00: 00: 55$ & $00: 00: 33$ & $00: 00: 36$ & $00: 04: 01$ & $00: 00: 26$ & $00: 00: 11$ \\
\hline 8 & $00: 00: 51$ & $00: 00: 40$ & $00: 00: 35$ & $00: 03: 49$ & $00: 00: 23$ & $00: 00: 12$ \\
\hline 8 & $00: 00: 54$ & $00: 00: 37$ & $00: 00: 37$ & $00: 03: 58$ & $00: 00: 25$ & $00: 00: 13$ \\
\hline 9 & $00: 00: 56$ & $00: 00: 39$ & $00: 00: 40$ & $00: 03: 41$ & $00: 00: 29$ & $00: 00: 10$ \\
\hline 9 & $00: 00: 53$ & $00: 00: 35$ & $00: 00: 39$ & $00: 03: 53$ & $00: 00: 28$ & $00: 00: 11$ \\
\hline 10 & $00: 00: 55$ & $00: 00: 34$ & $00: 00: 34$ & $00: 04: 05$ & $00: 00: 24$ & $00: 00: 15$ \\
\hline 10 & $00: 00: 57$ & $00: 00: 35$ & $00: 00: 37$ & $00: 03: 36$ & $00: 00: 26$ & $00: 00: 13$ \\
\hline
\end{tabular}

Fonte: Próprios autores.

Com os tempos dos processos em mãos foi utilizado a simulação computacional por meio do software ArenaversãoStudent 12, o modelo atual para simular o fluxo, descobrir a capacidade produtiva e identificar os processos críticos presentes no processo.

Afim de se obter uma amostra fidedigna dos tempos dos processos, foram elaboradosos gráficos de controle $\bar{x}$ e R no Minitab versão16, com o objetivo de 
verificar que os dados coletadosretratam a realidade da confecção não apresentando causas especiais. Laugeni e Martins (2010) recomendam o uso de gráfico de controle para analisar a qualidade dos dados.

Os gráficos de controle demostram as médias e as amplitudes (R). Neles os limites de controle são descritos como limite superior de controle (UCL) e limite inferior de controle (LCL).O quadro2 apresenta os limites de controle e a média dos tempos coletados.

Quadro2: Limite superior de controle (UCL), limite inferior de controle ( $L C L)$ e a média dos tempos coletados

\begin{tabular}{c|c|c|c|c|c|c|}
\cline { 2 - 6 } & Corte & Unir Frente & Unir Forro & $\begin{array}{c}\text { Unir Frente } \\
\text { e Forro }\end{array}$ & $\begin{array}{c}\text { Pregar } \\
\text { Elástico }\end{array}$ & Embalar \\
\hline $\mathrm{UCL}$ & $00: 00: 58$ & $00: 00: 40$ & $00: 00: 39$ & $00: 04: 11$ & $00: 00: 28$ & $00: 00: 15$ \\
\hline $\bar{x}$ & $00: 00: 55$ & $00: 00: 35$ & $00: 00: 36$ & $00: 03: 51$ & $00: 00: 25$ & $00: 00: 12$ \\
\hline $\mathrm{LCL}$ & $00: 00: 51$ & $00: 00: 30$ & $00: 00: 34$ & $00: 03: 29$ & $00: 00: 22$ & $00: 00: 09$ \\
\hline
\end{tabular}

Fonte: Próprios autores.

\section{RESULTADOS E DISCUSSÕES}

Partindo-se do contexto apresentado, desenvolveu-se um modelo de simulação computacional para a produção de máscaras. As simulações foram realizadas no Arena, considerando-se os recursos humanos, máquinas e equipamentos.

O tempo total da simulação da produção de máscaras foi de 480 minutos, pois equivale a um dia de trabalho com duração de 8 horas, tendo o início às $07 \mathrm{~h} 00 \mathrm{~min}$ e término às $17 \mathrm{~h} 00 \mathrm{~min}$, com um intervalo de 60 minutos para o almoço.Foram analisadas as variáveis: número de peças produzidas (Numberout), tempo médio em fila (Waiting Time) e taxa de utilização (InstantaneousUtilization) para as atividades modeladas.

O modelo elaborado foi apresentado ao gestor da confecção, sendo validado como uma representação real do sistema. A animação do software possibilitou uma melhor compreensão do fluxo produtivo, assim como, as suas 
deficiências. A figura 2 ilustra o modelo do processo produtivo adotado pela empresa para fabricação e máscaras.

Figura 2: Modelo do processo produtivo de máscaras elaborado através do softwareArena.

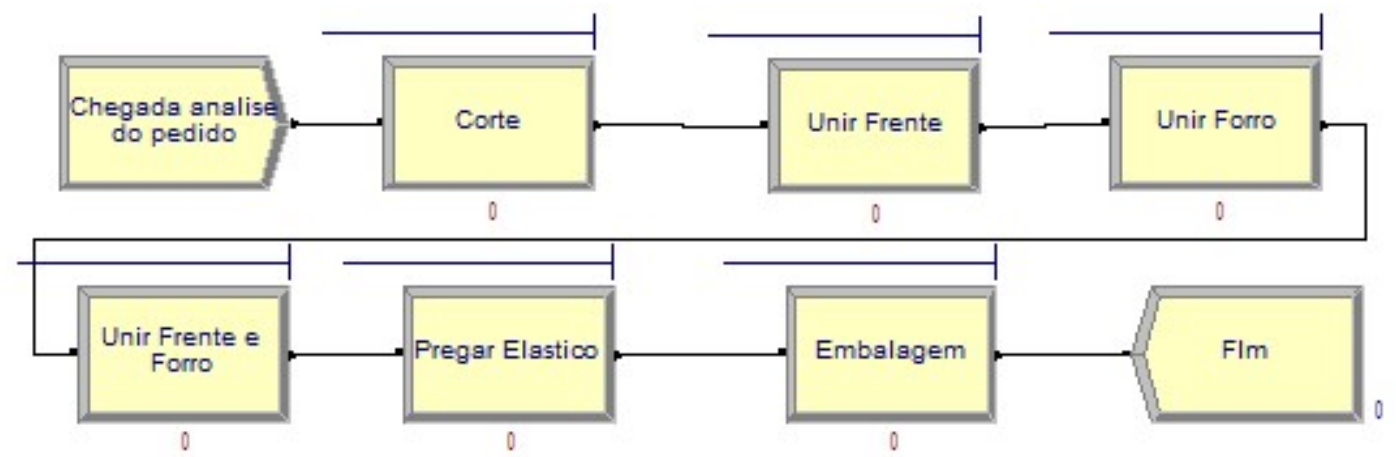

Fonte: Extraído do software Arena. Adaptado pelos autores.

O modelo diagnosticou que a fluxo produtivo tem uma deficiência de atender 14 máscaras. Acontece a entrada de 510 peças e somente é possível entregar 496 máscaras no final do dia de trabalho. A figura 3 ilustra o relatório extraído do software Arena.

Figura3: Performance do processo de fabricação

\begin{tabular}{|lr|}
\hline Number In & value \\
\hline Entity 1 & 510.00 \\
Number Out & value \\
\hline Entity 1 & 496.00 \\
\hline
\end{tabular}

Fonte: Extraído do software Arena. Adaptado pelos autores.

O modelo simulado apresentou gargalo em uma de suas etapas. O gargalo se restringiu somente no processo de unir frente e forro. A figura 4 apresenta 0 gargalo do decorrer do processo. 
Figura 4: Gargalo nos processos produtivos.

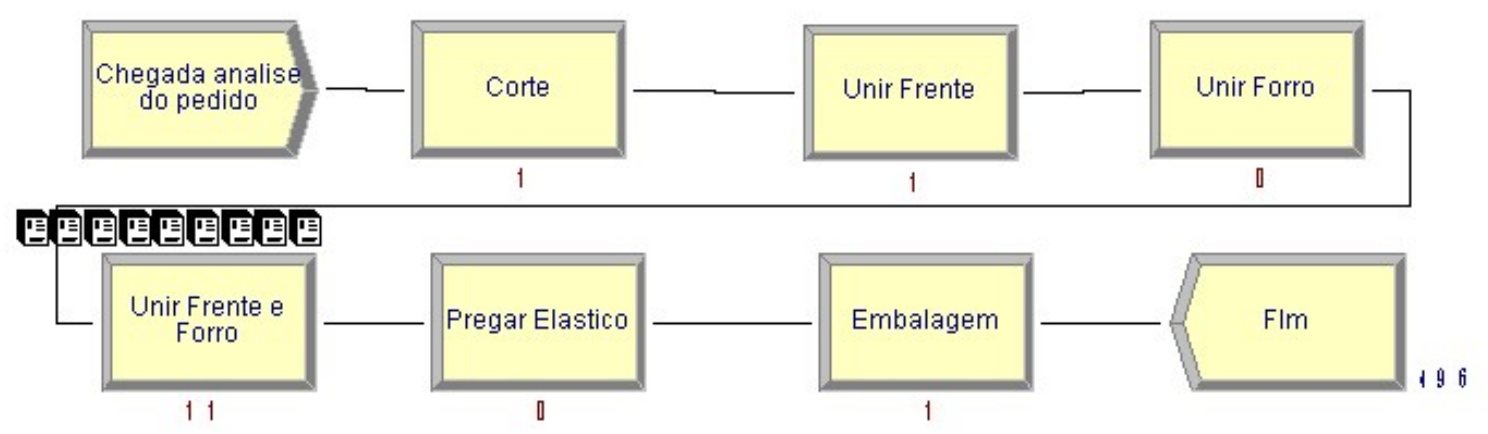

Fonte: Extraído do software Arena. Adaptado pelos autores.

O gargalo apresentaum valor mínimo de 294,80 segundos em espera na fila um valor máximo de 563,40 segundos. A figura 5 demostra o comportamento das filas no processo de fabricação em estudo.

Figura 5: Desempenho das filas nas etapas produtivas

\begin{tabular}{|lrrrr|}
\hline Waiting Time & Aversge & Half Width & $\begin{array}{r}\text { Minimum } \\
\text { Value }\end{array}$ & $\begin{array}{r}\text { Maximum } \\
\text { Value }\end{array}$ \\
\hline Corte.Queue & 0.00 & 0,000000000 & 0.00 & 0.00 \\
Embalagem.Queue & 0.00 & 0,000000000 & 0.00 & 0.00 \\
Pregar Elastico.Queue & 0.00 & 0,000000000 & 0.00 & 0.00 \\
Unir Forro.Queue & 0.00 & 0,000000000 & 0.00 & 0.00 \\
Unir Frente e Forro.Queue & 294.80 & (Correlated) & 0.00 & 563.40 \\
Unir Frente.Queue & 0.00 & 0,000000000 & 0.00 & 0.00 \\
\hline
\end{tabular}

Fonte: Extraído do software Arena. Adaptado pelos autores.

Através dos relatórios obtidos após a simulação, foi possível constatar à taxa de utilização do recurso para executar determinada atividade. Tendo como o processo de costurar frente e forro das máscaras como a maior taxa de ocupação ao logo do dia de trabalho $(99,65 \%)$, seguido pela etapa de costurar forro 
$(65,17 \%)$, costurar frente $(63,58 \%)$, cortar $(48,83 \%)$, costurar elástico $(43,61 \%)$ e embalar $(20,36 \%)$.Foi possível observar que a etapa de costurar frente e forro é a etapa mais crítica do processo, o colaborador costura as peças pelo lado avesso, no entanto, ao finalizar essa costura, demanda um tempo para virar o tecido para o lado direito, afim de concluir a costura, nessa parte do processo. A figura 6 apresenta os índices de ocupação de cada processo de fabricação.

Figura 6: Taxa de ocupação nas etapas produtivas.

\begin{tabular}{|lrrrr|}
\hline Instantaneous Utilization & Aversge & Half Width & $\begin{array}{r}\text { Minimum } \\
\text { Value }\end{array}$ & $\begin{array}{r}\text { Maximum } \\
\text { Value }\end{array}$ \\
\hline Cortar & 0.4883 & 0,004011738 & 0.00 & 1.0000 \\
Costurar elastico & 0.4361 & 0,004924488 & 0.00 & 1.0000 \\
Costurar forro & 0.6517 & 0,006818981 & 0.00 & 1.0000 \\
Costurar Frente & 0.6358 & 0,004967224 & 0.00 & 1.0000 \\
Costurar frente e forro & 0.9965 & (Correlated) & 0.00 & 1.0000 \\
Embalar & 0.2076 & 0,003653652 & 0.00 & 1.0000 \\
\hline
\end{tabular}

Fonte: Extraído do software Arena. Adaptado pelos autores.

De acordo com os dados analisados no tópico anterior, é possívelconstatar que há recursos com baixo nível de utilização. Como sugestão para tentar solucionar o problema da etapa do processo que é visto como gargalo, a confecção poderia usar operadores polivalentes, que executassem mais de uma tarefa, como por exemplo, combinando as atividades de embalar e auxiliar na etapa de costurar frente e forro. Uma segunda sugestão, seria utilizar o colaborador do setor de corte para também atuar no setor de embalagem, pois foram essas atividades que demonstraram um baixo nível de utilização.

\section{CONSIDERAÇÕES FINAIS}

O presente artigo procurou constatar por intermédiodas técnicas de simulação computacional no software Arena versãoStudent 12 a capacidade de produção do processo de fabricação de máscaras de tecido, seus gargalos e filas e o grau de ocupação dos recursos disponíveis. 
Por meio da simulação computacional feita através do softwareArenafoi possível replicar o modelo em que foi representado o dia a dia da empresa. A modelagem despertou para empresa a sua capacidade produtiva, gargalos e filas e o grau de ocupação do recurso.

O modelo constatou que o fluxo produtivo tem um déficitna sua produção, visto que, ocorre gargalos ao longo da linha produtiva e um desbalanceamento na utilização dos recursos.

De maneira a tentar solucionar o problema dos gargalos e balancear autilização dos recursos, a empresa poderia utilizar colaboradores polivalentes, que desenvolvessem mais de uma tarefa.

Diante disso, ressalta-se a importância da simulação computacional visando identificar a capacidade produtiva, a fim de extinguir possíveis perdas ao longo do processo.

\section{REFERÊNCIASBIBLIOGRÁFICAS}

ARAÚJO, C. A. L.; PEREIRA, C. F.A indústria de confecções em Pernambuco: impactos e oportunidades em um cenário pós-ATC (Acordo sobre Têxteis e Confecções). 2006. Disponível em: https://simpep.feb.unesp.br/anais/anais_13/artigos/233.pdf. Acesso em 26 de junho de 2020.

CAMPOS, K.C.F. Simulação em um Trecho Singelo de uma Malha Ferroviária utilizando o software Arena. 2006. Disponível em:

http://www.abepro.org.br/biblioteca/enegep2006_TR460314_7952.pdf. Acesso em 29 de maio de 2020.

FIORONI, M. M. Simulação em ciclo fechado de malhas ferroviárias e suas aplicações no Brasil: avaliação de alternativas para o direcionamento de composições. 2007. Disponível em: http://www.teses.usp.br/teses/disponiveis/3/3135/tde-03062008-180002/pt-br.php. Acesso em 14 de junho de 2020.

FREITAS FILHO, P J. Introdução à Modelagem e Simulação de Sistemas com Aplicações em Arena. Florianópolis: Visual Books, 2008.

GOMES, D. R. Mapeamento de Processos como ferramenta de avaliação de processo produtivo: Um estudo de caso em uma empresa do pólo de cerâmica de Campos - RJ. 2009. Disponível em: http://www.uenf.br/Uenf/Downloads/LEPROD_6975_1251810639.pdf. Acesso em 23 de junho de 2020.

GONÇALVES, A. A. Gestão da capacidade de atendimento em hospitais de câncer. 2004. Disponível em:

http://bvsms.saude.gov.br/bvs/publicacoes/inca/capa_tese_doutorado_Antonio_Augusto_2004.pdf . Acesso em 6 de junho de 2020. 
HARREL, C. R.; MOTT, J. R. A.; BATEMAN, R. E.; BOWDEN, R. G.; GOGG, T. J. Simulação: otimizando os sistemas. São Paulo: IMAM, 2002.

LAUGENI, F. P; MARTINS, P. G. Administração da Produção. São Paulo: Saraiva, 2010.

LIDÓRIO, C. F. Tecnologia da Confecção. Disponível em:

https://wiki.ifsc.edu.br/mediawiki/images/temp/5/52/20080729221515!Apostila_CTQ_edi\%C3\%A7

\%C3\%A3o_1.pdf. Acesso em 26 de junho de 2020.

MARTINS, A. G.; DOMINGUES, O. Estatística Geral e Aplicada. São Paulo: Editora Atlas, 2011.

OLIVEIRA, F. B. A simulação de uma central de operações e controle para emergências hospitalares em eventos de grande porte. 2012. Disponível em:

http://objdig.ufrj.br/60/teses/coppe_m/FabioBatistaDeOliveira.pdf. Acesso em 29 de maio de 2020.

PIAZZAROLLO, M, G.; OLIVEIRA, L. D.; LUZ, G. C.; SIMEÃO, I.Estudo de um layout por processo na indústria moveleira: um estudo de caso. Disponível em:

http://www.saepro.ufv.br/wp-content/uploads/2008-17.pdf. Acesso em 20 de junho de 2020.

REIS, C.C.C., NAUMANN, E. A., SCORTEGAGNA, C. Aplicação dos estudos de tempos em uma prestadora de serviços na busca do aumento da produtividade. 2015. Disponível em: http://www.abepro.org.br/biblioteca/TN_STP_206_220_27826.pdf. Acesso em 12 de junho de 2020.

SANTOS, J. A. A.; BEM, A. R. Modelagem e análise do processo de produção de uma indústria de mosaicos. Revista de Engenharia e Tecnologia. v. 5. n. 2. p. 170, 2013.

SILVA, C. A.; CALIXTO, W. P.; SANTOS, L. E. B.; MAGALHÃES, A. S.; ALVES, A. J.; DOMINGUES, E. G. Simulação das filas dos elevadores do Fórum da Cidade de Goiânia utilizando o Software Arena.Disponível em:

https://www.researchgate.net/publication/285420087_Simulacao_das_filas_dos_elevadores_do_F orum_da_Cidade_de_Goiania_utilizando_o_Software_Arena?channel=doi\&linkld=565e235f08ae 1 ef92983aee6\&showFulltext=true. Acesso em 12 de junho de 2020.

SILVA, L. M. F.; P, M. G.; SUBRAMANIAN, A. Utilizando o software Arena como Ferramenta de Apoio ao Ensino em Engenharia de Produção. 2013. Disponível em:

http://www.abepro.org.br/biblioteca/ENEGEP2012_TN_WIC_157_918_20123.pdf. Acesso em 13 de junho de 2020. 